\title{
Effect of the number of vortices on the torque scaling in Taylor-Couette flow
}

\author{
B. Martínez-Arias, J. Peixinho†, O. Crumeyrolle and I. Mutabazi \\ Laboratoire Ondes et Milieux Complexes, Université du Havre \& CNRS UMR 6294, \\ 53 rue de Prony, 76600 Le Havre, France
}

(Received 27 May 2022)

\begin{abstract}
Torque measurements in Taylor-Couette flow, with large radius ratio and large aspect ratio, over a range of velocities up to a Reynolds number of 24000 are presented. Following a specific procedure, nine states with distinct number of vortices along the axis were found and the aspect ratio of the vortices were measured. The relationship between the speed and the torque for a given number of vortices is reported. In the turbulent Taylor vortex flow regime, at relatively high Reynolds number, a change in behaviour is observed corresponding to intersections of the torque-speed curves for different states. Before each intersection, the torque for a state with larger number of vortices is higher. After each intersection, the torque for a state with larger number of vortices is lower. The exponent, from the scaling laws of the torque, always depends on the aspect ratio of the vortices. When the Reynolds number is rescaled using the mean aspect ratio of the vortices, only a partial collapse of the exponent data is found.
\end{abstract}

\section{Introduction}

Since the works by Mallock (1888), Couette (1890), Taylor (1936) and Wendt (1933), there was theoretical and experimental interest in the torque from liquids confined between two concentric differentially rotating cylinders.

Following the work by Taylor (1923), important contributions in the understanding of this flow instability have been made by many authors, including Stuart (1958), Donnelly \& Simon (1960), Coles (1965), Snyder (1969), Gollub \& Swinney (1975), Barcilon et al. (1979), Koschmieder (1979), Mullin \& Benjamin (1980), Di Prima \& Swinney (1981), Benjamin \& Mullin (1982), Nakabayashi et al. (1982), Riecke \& Paap (1986), Cliffe et al. (1992), Lathrop et al. (1992), Lewis \& Swinney (1999), Takedal(1999), Xiao et al. (2002), Czarny et al. (2003), Abshagen et al. (2005), Lim \& Tan (2004), Racina \& Kind (2006), Burin et al.|(2010), Dutcher \& Muller (2009) and many others. Because of these successes, the Taylor-Couette system is used to investigate the turbulence scaling laws, especially the behaviour of dimensionless torque acting on the rotating cylinder as a function of the Reynolds number.

From a practical point of view, Wendt (1933) and Donnelly \& Simon (1960) provided scaling laws of the dimensionless torque, $G$, as a function of Reynolds number, Re, i.e. $G \propto R e^{\alpha}$ with an exponent, $\alpha$, that depends on $R e$ and other parameters. More recent studies (Lathrop et al. 1992, Lewis \& Swinney 1999; Dubrulle et al. 2005; Ravelet et al. 2010; Paoletti \& Lathrop 2011:|van Gils et al.|2011) provided additional data on torque and were concerned with fairly larger Reynolds numbers.

$\dagger$ Email address for correspondence: jorge.peixinho@univ-lehavre.fr 
Eckhardt et al. (2007) proposed analogies between turbulence in Rayleigh-Bénard convection and Taylor-Couette flow with emphasis on the evolution of the exponent. An argument was put forward to investigate turbulent Taylor-Couette flow because it has a stronger driving than Rayleigh-Bénard flow and should allow access to the ultimate turbulent regime (Huisman et al. 2012, Ostilla-Mónico et al. 2014). This unifying theory was tested using the data of Lathrop et al. (1992) and Lewis \& Swinney (1999). They found a good agreement for a range of Reynolds above $10^{4}$.

From a numerical point of view, several groups (Coughlin \& Marcus 1996: Batten et al. 2002; Bilson \& Bremhorst 2007; Pirró \& Quadrio 2008; Brauckmann \& Eckhardt |2013, Ostilla et al. 2013 Ostilla-Mónico et al.|2014) were able to simulate numerically turbulent Taylor-Couette flow up to relatively high Re. Most of these investigations (Brauckmann \& Eckhardt 2013 Ostilla et al. 2013; Ostilla-Mónico et al. 2014) use periodic boundary conditions with relatively short calculation domains. Ostilla et al. (2013) presented the effect of three and four vortex pairs on the dimensionless transport suggesting that the larger number of vortex pairs induces an increase in torque. Brauckmann \& Eckhardt (2013) investigated the effect of the vortex size on the torque and found a maximum of torque for vortices of axial wavelength of 1.93 times the gap width for $R e=5000$. Hence, the Taylor-Couette system offers an opportunity to vary the number of vortices for a fixed value of the aspect ratio and therefore to modify the vortices shape and the shear between two adjacent vortices. Yet, all the recent studies do not explicitly mention the number of cells, although both Lathrop et al. (1992) and Lewis \& Swinney (1999) provided torque data for eight and ten vortex states.

In this article, we elucidate the effect of the number of vortices on the torque-speed relationship in a system containing up to nine states. This article is organised as follows. A description of the experimental apparatus is given in $\S 2$ together with a detailed discussion of the protocols to obtain the different number of vortices. This is followed, in $\S 3$, by the results on the torque data which are analysed. Finally, we draw some conclusions in $\S 4$.

\section{Experimental setup and procedure}

\subsection{Experimental setup}

The Taylor-Couette geometry used here is fitted on a rheometer (Physica MCR 501, Anton Paar). Figure 1 shows a sketch of the experiment. The inner cylinder has a radius $r_{i}=50 \pm 0.01 \mathrm{~mm}$ and the outer cylinder $r_{o}=55 \pm 0.01 \mathrm{~mm}$. Hence, the gap between both cylinder is $d=r_{o}-r_{i}=5 \pm 0.01 \mathrm{~mm}$. The length of the inner cylinder is $L=150 \pm 0.5 \mathrm{~mm}$. Consequently, the dimensionless parameters which describe the geometry are the radius ratio $\eta=r_{i} / r_{o}=0.909$ and the aspect ratio $\Gamma=L / d=30$. The object of the present experiments was to examine the relationship between the angular speed of the inner cylinder, $\Omega$, and the torque that it exerts on the fluid, $T$. The Reynolds number, $R e$, is based on the angular velocity of the inner cylinder, the inner cylinder radius, the gap between cylinders and the properties of the fluid: $R e=\Omega r_{i} d / \nu$, where $\nu$ is the kinematic viscosity of the working fluid. The dimensionless torque, $G$, is based on the torque exerted by the fluid in the walls of the inner cylinder, the height of the inner cylinder and the properties of the fluid. $G$ is defined as $G=T / 2 \pi \rho \nu^{2} L$, where $\rho$ is the density of the working fluid. In addition to $G$, the so-called $\omega$-Nusselt number, $N u_{\omega}$, defined according to Eckhardt et al. (2007), is also used:

$$
N u_{\omega}=\frac{G}{G_{l a m}}, \quad \text { where } \quad G_{l a m}=\frac{2 \eta}{(1+\eta)(1-\eta)^{2}} R e
$$




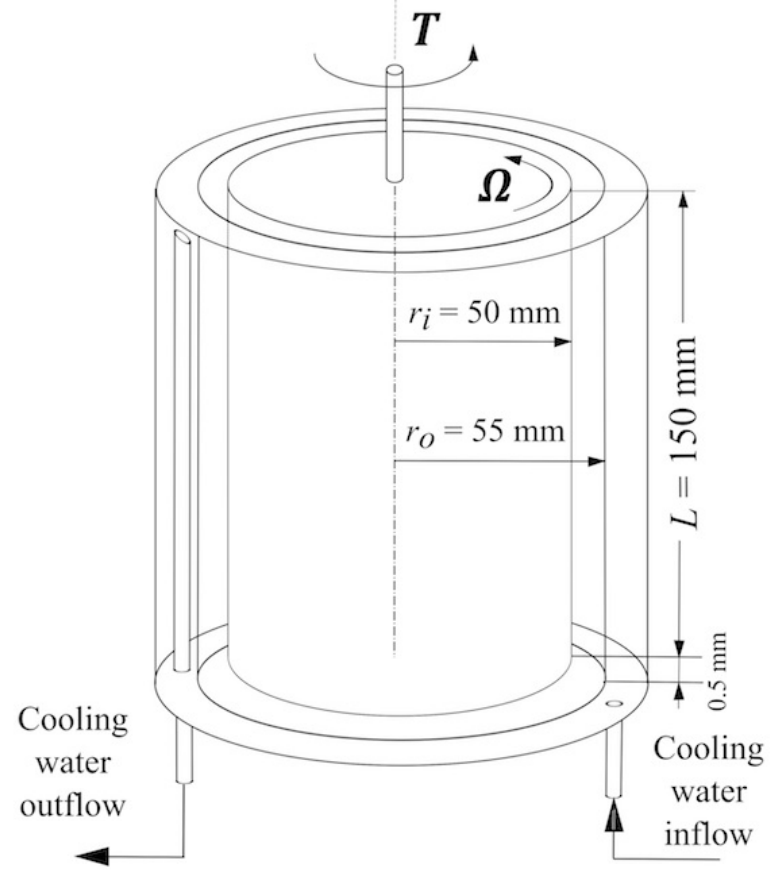

FigurE 1. Sketch of the Taylor-Couette system, drawn to scale.

$N u_{\omega}$ represents the torque measured in the units of laminar torque.

The inner cylinder is made of aluminum and its surface is anodized. The bottom of the inner cylinder is recessed. There is a gap of $0.5 \pm 0.001 \mathrm{~mm}$ between the edge of the base of the inner cylinder and the flat bottom of the outer cylinder filled with an air bubble, which minimises the shear stress on the bottom of the cylinder. The top part of the gap between both cylinders is covered with an annular PVC lid. It is positioned so that the bottom of the lid is at the same height as the upper edge of the inner cylinder. That makes the gap being completely filled and there is no contact between the lid and the inner cylinder. The outer cylinder is made of glass and there is an additional glass jacket connected to a flow of water in order to maintain the working temperature at $22 \pm 0.01^{\circ} \mathrm{C}$. For flow visualization purposes, $2 \%$ of Kalliroscope is added to the fluid. The rheometer allows for torque or speed controlled runs. The highest acquisition frequency is $100 \mathrm{~Hz}$ and the real resolution of the encoder is smaller than $1 \mu \mathrm{rad}$. The accuracy of the torque is $0.5 \%$ of the measured value and never smaller than $0.2 \mu \mathrm{Nm}$. Several fluids, mixtures of water and glycerol, were used in order to optimize the speed acquisition. Additional measurements were done using a low viscosity silicon oil in order to reach data in the highest range of $R e$.

\subsection{Experimental procedure}

In quasi-static ramping of the velocity, the Taylor vortex flow is characterised by 30 time-independent, axisymmetric, toroidal vortices from $R e_{c}=138$. This value is close to the predicted value (139) given by the stability theory (Esser \& Grossmann 1996). For $157<R e<199$, wavy vortex flow is seen. Then, from $R e=199$, modulated wavy vortex is observed.

In figure 2, a spatio-temporal diagram over the whole height of the cylinders is presented. The flow is driven by the rotation of the inner cylinder at a constant ramping 


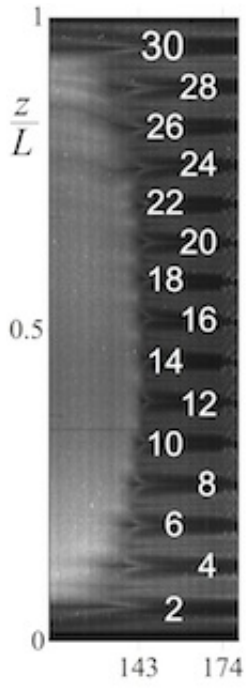

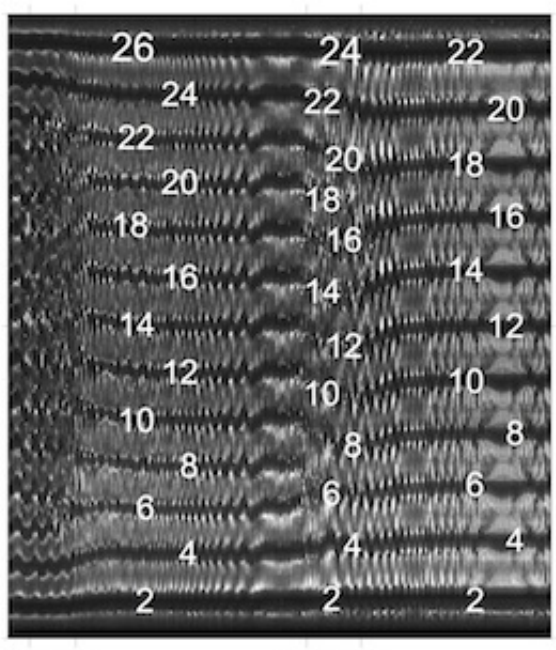

360380

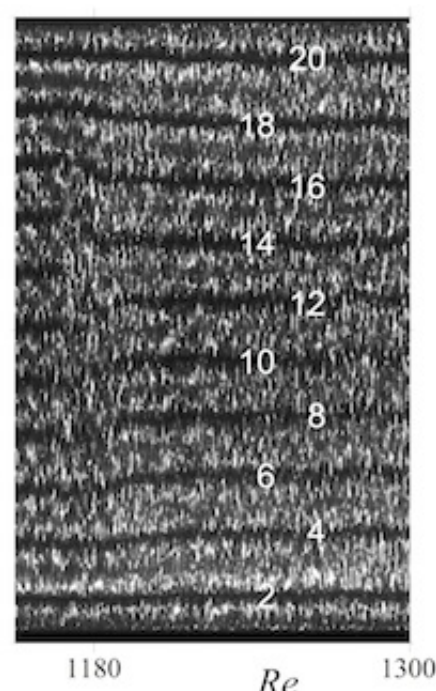

Re

Figure 2. Spatio-temporal diagram over the whole height $(\Gamma=L / d=30)$ of the Taylor-Couette flow $(\eta=0.909)$ in three intervals from $R e=110$ to 1300 . The acceleration rate is : $\partial R e / \partial \tau=4$. The numbers on the diagram count the number of cells from bottom to top.

rate: $\partial R e / \partial \tau=4$, where $\tau=t \nu / d^{2}$ and $t$ is time. As $R e$ increases from the laminar Couette flow, Eckman vortices develop at the ends of the cylinders (Czarny et al. 2003). Then these vortices evolve into a well defined state of axisymmetric steady toroidal Taylor vortices that rapidly join at the center of the cylinder at $R e=143$. At $R e=174$, the wavy vortex flow starts. In figure 2 , the second interval $(250<R e<450)$ shows sequences of regions of strong modulation leading to the merging of cells $(26,24$ and 22 ). In this same range of $R e$, Coles (1965) showed in a system of similar aspect ratio that a large number of expected states were accessible due to the vertical oscillations and the merging of these cells. Clearly, as we progress in time or in Re the number of cells decreases from 30 to 20 . To obtain a smaller number of cells, say 18, a lower acceleration is used. Hence, this merging of cells will allow to prepare an initial state with 30 to 18 cells. Only the number of cells is taken into account and spiral modes are not considered here. Once the desired number of cells is set up, our strategy is to change instantaneously the torque to a prescribed value and then measure the velocity for few minutes before the next measurement. The stability of each state was tested during approximately 70 times the viscous time, i.e. $\nu / d^{2}$, to ensure that the number of vortices remained constant. The velocity fluctuations of the rotating cylinder are small, typically around $0.6 \%$.

\section{Results and discussion}

The results are a combination of torque measurements and simultaneous flow visualisations. The first set of results is concerned with the properties of two distinct states: 30 and 18 cells states. The second set of results presents and analyses the torque data, describes the stability limit of the states as well as the change in behaviour in the torque-speed curves for different states.

\subsection{Aspect ratio of cells}

The finding of different states for the same boundary conditions requires that the size of the cells varies from one state to the other. In figure $3(a)$ and $(b)$, photographs of 
(a)

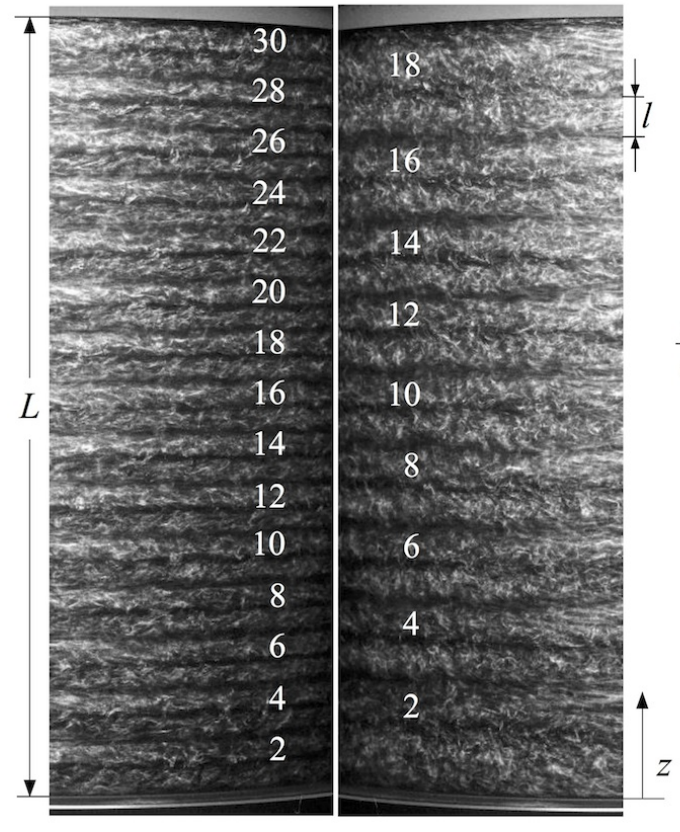

(c)

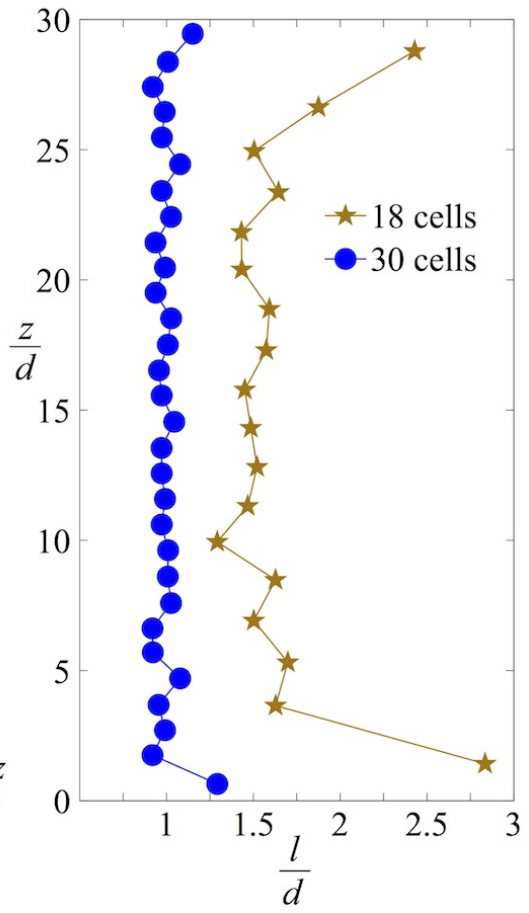

FigURE 3. Photographs and aspect ratio along the axis of 30 and 18 cellular modes at $R e=6000$. (a) Normal 30 cells, (b) abnormal 18 cells, and (c) the associated aspect ratio along the axis.

two states with 30 and 18 cells at $R e=6000$ are presented. Figure $3(c)$ presents their aspect ratio along the vertical axis. For intermediate number of cells: 20, 22, 24, 26 and 28 , intermediate curves are expected. For the primary state of 30 cells, the aspect ratio, $l / d$, of all the cells is close to one. However for the 18 cells pattern, the ratio $l / d$ is 1.5 in the center of the cylinder so the cells are elongated. Note the cells close to the ends of the cylinders have a significant larger aspect ratio up to 2.5. This indicates that the caps have a local effect and strongly elongate the two cells close to the ends (Czarny et al. 2003). Although the data on the aspect ratio are for $R e=6000$, using the same protocol, we have observed states with different numbers of vortices over a wide range of $R e$, that looks essentially similar to the ones in figure $3(a, b)$.

The states in figure 3 were obtained at $R e=6000$. The difference in the measured torque between the 18 cells state and the 30 cells state is $13.5 \%$. The state with the larger number of cells experiences a larger torque. This is in agreement with the numerical simulation of Ostilla et al. (2013) although their range of $R e(400<R e<1600)$ and the number of cells tested ( 6 or 8 cells) is smaller.

\subsection{Torque}

Torque and speed measurements have been performed up to a $R e$ of 24000 . Using the procedure presented earlier we were able to measure the torque associated to 7 different states: 30, 28, 26, 24, 22, 20 and 18 cells. The relationship between the $\omega$-Nusselt number and the Reynolds number for these states is presented in figure 4 . Different symbols and colors represent the different states: the laminar Couette flow, the Taylor vortex flow (30 cells), the wavy vortex flow and the 7 different turbulent Taylor vortex flows with 30, 28, 


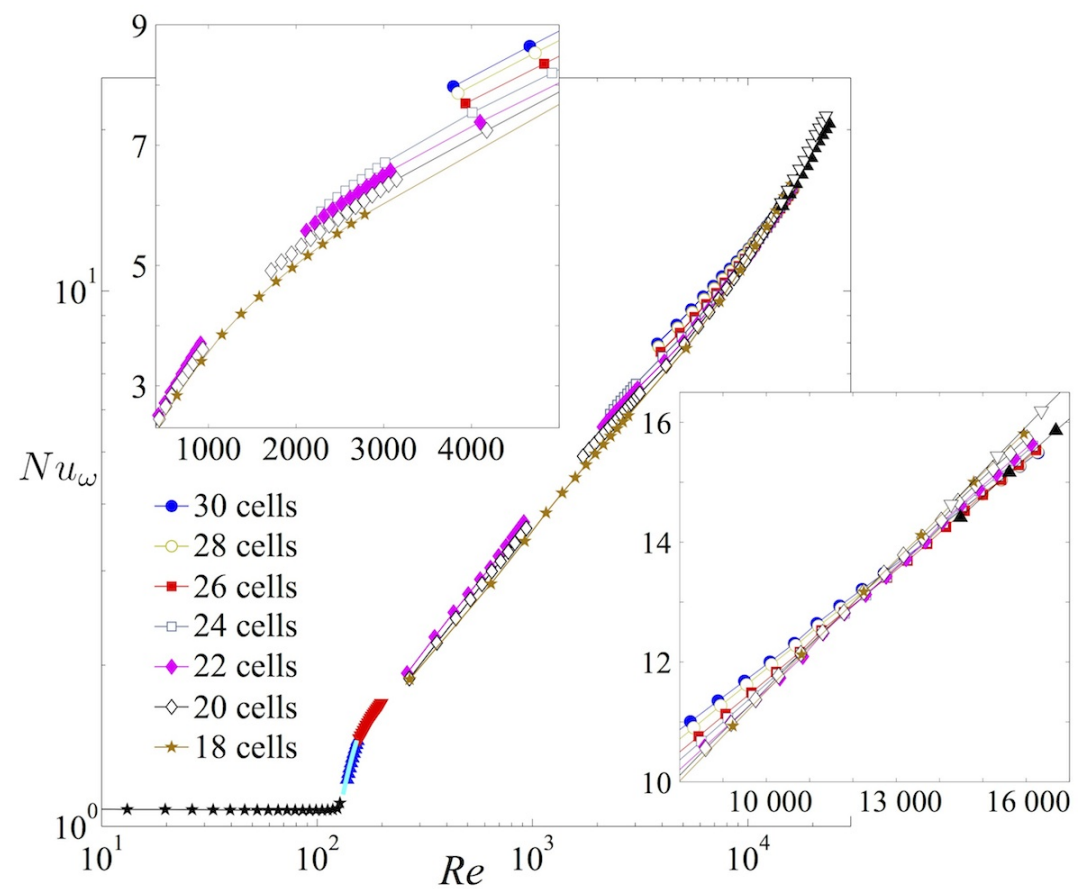

Figure 4 . Rescaled torque, $N u_{\omega}$, as a function of $R e$ for the different flow states. The black stars $(\star)$, the blue triangles $(\boldsymbol{\Delta})$ and the red triangles $(\boldsymbol{\nabla})$ represent the Couette flow, the Taylor vortex flow and the wavy vortex flow, respectively. The thick (cyan) line represents a fitting using the proposed scaling of Donnelly \& Simon (1960). Top inset is a zoom at small Re in linear scale showing the stability limit of some states. Bottom inset is a zoom at large $R e$ in linear scale showing the intersections. The black triangles $(\boldsymbol{\Delta})$ and the empty triangles $(\nabla)$ represent data obtained using low viscosity silicone oil. The error bars are smaller than the plotting symbols.

26, 24, 22, 20 and 18 cells. In order to access large values of Re up to 24 000, low viscosity silicone oil was used and two series of data are reported in figure 4 . The use of silicone oil does not allow to visualise the flow and therefore to count the number of vortices. These data were obtained by applying protocols leading to states with large and small number of cells. The trend of the curves suggests that the black triangles correspond to large number of cells and empty triangles to small number of cells.

In figure 4, the $\omega$-Nusselt number associated to Couette flow $\left(R e<R e_{c}\right)$ is almost constant and just above one; the small difference from one is due to end effects. The Taylor vortex flow and the wavy vortex flow have distinct properties, corresponding to different slopes in the $N u_{\omega}$ versus $R e$ curve. The rescaled torque behaviour for Taylor vortex flow $(138<R e<154)$ is fitted by a relation suggested from the finite amplitude theory by Stuart (1958) and proposed by Donnelly \& Simon (1960):

$$
N u_{\omega}=a R e^{-2}+b R e^{0.36}
$$

with $a=-13374$ and $b=0.33$.

The stability domain of the different number of vortices is in the top inset of figure 4 It is interesting to notice that the 30 , the 28 and the 26 vortices states are not stable for Re below 3800. Similarly, the 24, the 22 and the 20 cells states are not stable for $R e$ below 2300, 2100 and 1700, respectively. In the range between 1000 and 1700 , only the 18 cells state is stable. These results are reminiscent of the stability studies from Coles (1965), Snyder (1969), Koschmieder (1979) and Cliffe et al. (1992). 
As the Reynolds number increases, a systematic increase of $\omega$-Nusselt is observed. The 18 cells state has always the lowest $\omega$-Nusselt whereas the states associated to the largest number of cells have always the highest $\omega$-Nusselt. As the Reynolds number increases further the curves $N u_{\omega}$ versus $R e$ come closer. Then, the curves for different states intersect in a range of $R e$ between 9600 and 15 500. A zoom in this range is shown in the bottom inset of figure 4 where most of the intersections are located around 12600 . Below each intersection, the torque is larger for states where the number of cells is larger. Above each intersection, the trend changes and a smaller number of cells leads to larger torque. Similar intersections were reported by Lathrop et al. (1992) and Lewis \& Swinney (1999) for eight and ten cells states in a system with a radius ratio of 0.724 .

The analysis of our data allows to present, in figure 5, the rescaled torque as a function of the aspect ratio of the vortices, $\bar{l} / d$, for several values of $R e$. For relatively small $R e$, the rescaled torque decreases as the aspect ratio of the vortices increases. For large $R e$, the rescaled torque increases with $\bar{l} / d$. At $R e=13000, N u_{\omega}$ is almost constant. In the experiments, $\bar{l} / d$ between 0.88 to 1.5 correspond to states with 34 to 18 cells. Our results confirm a clear effect of the vortex aspect ratio on $N u_{\omega}$, which was predicted by numerical simulations (Brauckmann \& Eckhardt 2013, Ostilla et al. 2013). For $R e=5000$, our values of $N u_{\omega}$ are slightly lower than the numerical results of Brauckmann \& Eckhardt (2013) and do not exhibit a peak that these authors found in their data at $\bar{l} / d=0.965$. The discrepancy between the experiments and the numerical simulations may be due to the fact that the numerical simulations were performed for a radius ratio of 0.71 .

\subsection{Exponent}

The dependence of $N u_{\omega}$ on Re can be described by

$$
N u_{\omega}=\mathcal{A} R e^{\alpha-1},
$$

where $\mathcal{A}$ depends on the vortex aspect ratio and is decreasing function of Re. A motivation behind seeking scaling laws is that insight into the turbulence mechanisms can be uncovered (see Dubrulle et al. 2005, Eckhardt et al. 2007). It is clear that the scaling laws discussed above cannot hold for Taylor vortex flow and wavy vortex flow since the flow is non-turbulent. Donnelly \& Simon (1960) discussed this issue and proposed the scaling (3.1) for Taylor vortex flow. For the range of Re below 3000, the exponent found in the present study is almost constant $(\alpha \simeq 1.5)$ and agrees well with the data from Lim $\& \operatorname{Tan}(2004)$ and Ravelet et al. (2010).

Figure 6(a) displays the variation of $\alpha-1$ as a function of $R e$ for different number of cells for $4000 \lesssim R e \lesssim 21000$. Here, $\alpha-1=\partial\left(\log _{10} N u_{\omega}\right) / \partial\left(\log _{10} R e\right)$ is calculated for each state separately as a function of the Re using a sliding least square fit (Lathrop

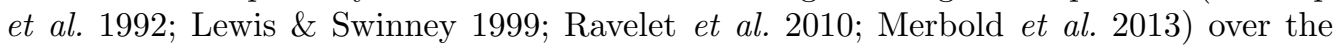
interval $\Delta\left(\log _{10} R e\right)=\Delta_{10}=0.1$. For the 18 cells state the interval is 0.2 . The results are compared with Ravelet et al. (2010) whose experiment has $\eta=0.917$ and $\Gamma=22$. Although, their aspect ratio is smaller then ours, there is a good agreement between their results and the present exponents for the 20 and 22 cells states. Both studies exhibit monotonous increase for $3000 \lesssim R e \lesssim 15000$.

A new scaling of the Reynolds number based on the mean aspect ratio of the cells, $\bar{l} R e / d$, is proposed for the exponent in figure $6(b)$. Only a partial collapse of the data is found. This suggests that the size of the cells is an important parameter in the turbulent regime studied here. Note that at higher $\bar{l} R e / d$, the collapse is better.

The meaning of $\alpha$ is related to the viscosity dependence of the torque (Lathrop et al. 1992). The Kolmogorov assumption assumes $\alpha=2$ for fully developed turbulence (Doering \& Constantin 1992). Any deviation from $\alpha=2$ implies a particular form of velocity 


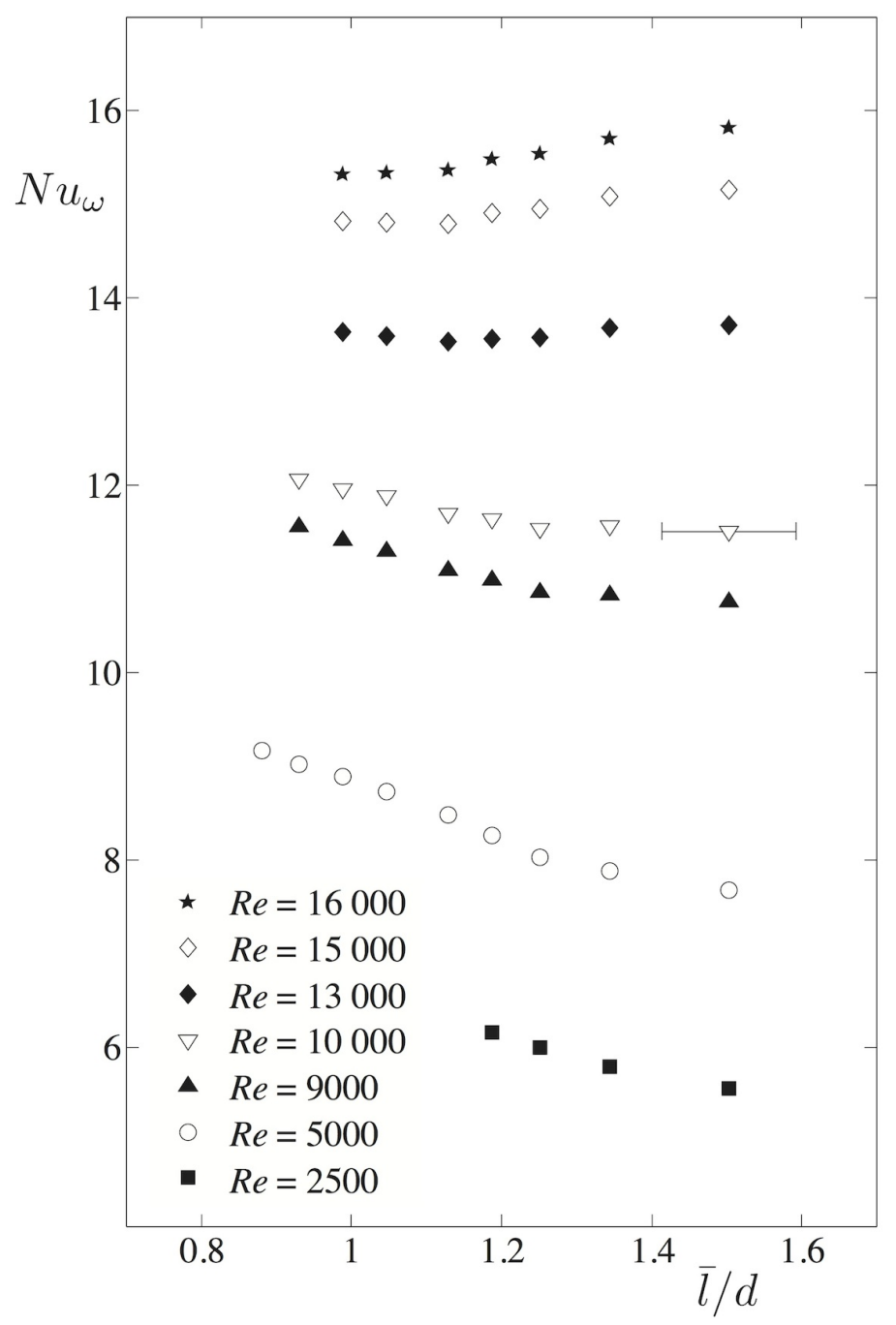

FiguRE 5. The rescaled torque in dependence on the aspect ratio of the cells for different $R e$. The horizontal error bar for $R e=10000$ represents the maximum error on $\bar{l}$. The vertical error on $N u_{\omega}$ is smaller than the symbol height.

fluctuations. In the flow system with a given number of vortices, the form of velocity fluctuations is constrained to the presence of large scale vortices and cannot be completely random. Modern investigations using particle image velocimetry (see Racina \& Kind 2006: Tokgoz et al. 2012) aim at quantifying the velocity fluctuations and estimating the average turbulent kinetic energy dissipation rate.

The principal feature, we wish to highlight, is the systematic dependence of the scaling on the aspect ratio of the cells, displayed in figure 6. For situations where the number of cells is small, typically 18 here, the flow is less constrained by the cells. Therefore, the turbulent flow will exhibit larger velocity fluctuations leading to higher turbulence and a larger $\alpha$. For situations were the number of cells is large, typically 30 , the flow is more constrained by the cells and the velocity fluctuations are weaker leading to a smaller $\alpha$. 

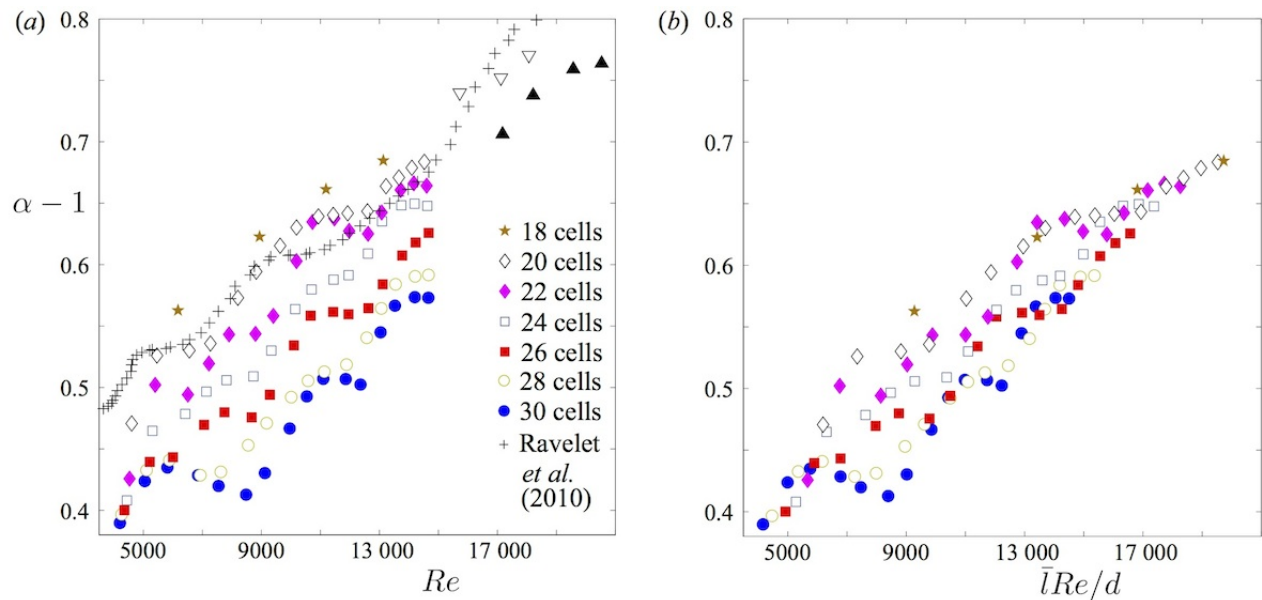

FiguRE 6. Evolution of the exponent for different number of cells. The black triangles $(\boldsymbol{\Delta})$ and the empty triangles $(\nabla)$ represent data obtained using low viscosity silicone oil. $(a) \alpha-1$ as a function of $R$ e and $(b) \alpha-1$ versus $\bar{l} R e / d$, based on the averaged height of the cells, $\bar{l}$

\section{Conclusions}

The effect of the number of vortices on torque in a Taylor-Couette flow $(\eta=0.909$ and $\Gamma=30)$ up to $R e=24000$ has been quantified. A specific protocol was used to obtain different states with $34,32,30,28,26,24,22,20$ and 18 cells. The evolution of the rescaled torque, $N u_{\omega}$, versus $R e$ for different number of cells is obtained. The results are in agreement with Lim \& Tan (2004) and Ravelet et al. (2010) and, moreover, the curves superpose self-consistently.

The effect of the vortex size on the rescaled torque indicates a change in behaviour corresponding to the intersection in the $N u_{\omega}$ versus $R e$ curves at $R e$ between 9600 and 15 500. For most of the states, the intersection is around 12 600. Before each intersection, the torque is larger for large number of cells and smaller after the intersection. In the same range of $R e$, but for a system with radius ratio of 0.725 , Lathrop et al. (1992) found a transition from centrifugal instability to shear turbulence using torque and local wall shear stress data. Lewis \& Swinney (1999) confirmed this transition by means of additional data of torque and wall shear stress, as well as velocity measurements. The data from Lewis \& Swinney (1999) shows a similar intersection for eight and ten cells states. In our case, it is not possible to conclude that the intersection is an indicator of the transition to shear-driven turbulence.

The scaling exponent of the torque is larger for states with large aspect ratio. It is also found that the exponents collapse when scaled with a Reynolds number based on the aspect ratio of the vortices. Finally, new large experimental apparatuses have been built (van Gils et al. 2011; Merbold et al. 2013, Avila \& Hof 2013) and these effects will be further detailed in ranges of higher $R e$ if the future investigations dare count the number of vortices.

We are grateful to Florent Ravelet for providing the data from Ravelet et al. (2010) and Sebastian Merbold for discussions on the calculation of the exponents. The authors also acknowledge the financial support of the Région Haute Normandie and the Agence Nationale de la Recherche (ANR), through the program "Investissement d'Avenir" (ANR10-LABX-09-01), LabEx EMC3. 


\section{REFERENCES}

Abshagen, J., Lopez, J. M., Marques, F. \& Pfisher, G. 2005 Mode competition of rotating waves in reflection-symmetric Taylor-Couette flow. J. Fluid Mech. 540, 269-299.

AvilA, K. \& Hof, B. 2013 High-precision Taylor-Couette experiment to study subcritical transitions and the role of boundary conditions and size effects. Rev. Sci. Instrum. 84, 065106.

Barcilon, A., Brindley, J., Lessen, M. \& Mobbs, F. R. 1979 Marginal instability in TaylorCouette flows at a very high Taylor number. J. Fluid Mech. 94, 453-463.

Batten, W. M. J., Bressloff, N. W. \& Turnock, S. R. 2002 Transition from vortex to wall driven turbulence production in the Taylor-Couette system with a rotating inner cylinder. Int. J. Numer. Meth. Fluids 38 (3), 207-226.

Benjamin, T. B. \& Mullin, T. 1982 Notes on the multiplicity of flows in the Taylor experiment. J. Fluid Mech. 121, 219-230.

Bilson, M. \& Bremhorst, K. 2007 Direct numerical simulation of turbulent Taylor-Couette flow. J. Fluid Mech. 579, 227-270.

Brauckmann, H. J. \& Eckhardt, B. 2013 Direct numerical simulation of local and global torque in Taylor-Couette flow up to $R \mathrm{e}=30$ 000. J. Fluid Mech. 718, 398-427.

Burin, M. J., Schartman, E. \& Ji, H. 2010 Local measurements of turbulent angular momentum transport in circular Couette flow. Exp. Fluids 48, 763-769.

Cliffe, K. A., Kobine, J. J. \& Mullin, T. 1992 The role of anomalous modes in TaylorCouette flow. Proc. Roy. Soc. Lond. A 439, 341-357.

Coles, D. 1965 Transition in circular Couette flow. J. Fluid Mech. 21, 385-425.

Couette, M. 1890 Etudes sur le frottement des liquides. Annal. Chim. Phys. 6, 433-510.

Coughlin, K. \& Marcus, P. S. 1996 Turbulent bursts in Couette-Taylor flow. Phys. Rev. Lett. 77, 2214-2217.

Czarny, O., Serre, E., Bontoux, P. \& Lueptow, R. M. 2003 Interaction between Ekman pumping and centrifugal instability in Taylor-Couette flow. Phys. Fluids 15, 467-477.

Di Prima, R. C \& Swinney, H. L. 1981 Instabilities and transition in flow between concentric rotating cylinders. In Hydrodynamics Instabilities and the transition to turbulence, Topics in Applied Physics, vol. 45, pp. 139-180. Springer.

Doering, C. R. \& Constantin, P. 1992 Energy equation in shear driven turbulence. Phys. Rev. Lett. 69, 1648-1651.

Donnelly, R. J. \& Simon, N. J. 1960 An empirical torque relation for supercritical flow between rotating cylinders. J. Fluid Mech. 7, 401-418.

Dubrulle, B., Dauchot, O., Daviaud, F., Longaretti, P.-Y., Richard, D. \& Zahn, J.-P. 2005 Stability and turbulent transport in Taylor-Couette flow from analysis of experimental data. Phys. Fluids 17, 95103.

Dutcher, C. S. \& Muller, S. 2009 Spatio-temporal mode dynamics and higher order transitions in high aspect ratio newtonian Taylor-Couette flows. J. Fluid Mech. 641, 85-113.

Eckhardt, B., Grossmann, S. \& Lohse, D. 2007 Torque scaling in turbulent Taylor-Couette flow between independently rotating cylinders. J. Fluid Mech. 581, 221-250.

Esser, A. \& Grossmann, S. 1996 Analytic expression for Taylor-Couette stability boundary. Phys. Fluids 8, 1814-1819.

Gollub, J. P. \& Swinney, H. L. 1975 Onset of turbulence in a rotating fluid. Phys. Rev. Lett. 35 (14), 927-930.

Huisman, S. G., van Gils, D. P. M., Grossmann, S., Sun, C. \& Lohse, D. 2012 Ultimate turbulent Taylor-Couette flow. Phys. Rev. Lett. 108, 24501.

Koschmieder, E. L. 1979 Turbulent Taylor vortex flow. J. Fluid Mech. 93, 515-527.

Lathrop, D. P., Fineberg, J. \& Swinney, H. L. 1992 Transition to shear-driven turbulence in Couette-Taylor flow. Phys. Rev. A 46, 6390-6405.

Lewis, G. S. \& Swinney, H. L. 1999 Velocity structure functions, scaling and transitions in high-Reynolds-number Couette-Taylor flow. Phys. Rev. E 59, 5457-5467.

Lim, T. T. \& TAN, K. S. 2004 A note on power-law scaling in a Taylor-Couette flow. Phys. Fluids 16, 140-144.

Mallock, A. 1888 Determination of the viscosity of water. Proc. R. Soc. Lond. 45, 126-132.

Merbold, S., Brauckmann, H. J. \& Egbers, C. 2013 Torque measurements and numerical 
determination in differentially rotating wide gap Taylor-Couette flow. Phys. Rev. E 87, 23014.

Mullin, T. \& Benjamin, T. B. 1980 Transition to oscillatory motion in the Taylor experiment. Nature 288, 567-659.

NAKabayashi, K., Yamada, Y. \& Kishimoto, T. 1982 Viscous frictional torque in the flow between two concentric rough cylinders. J. Fluid Mech. 119, 409-422.

Ostilla, R., Stevens, R. J. A. M., Grossmann, S., Verzicco, R. \& Lohse, D. 2013 Optimal Taylor-Couette flow: direct numerical simulation. J. Fluid Mech. 719, 14-46.

Ostilla-Mónico, R., van der Poel, E. P., Verzicco, R., Grossmann, S. \& Lohse, D. 2014 Boundary layer dynamics at the transition between the classical and the ultimate regime of taylor-couette flow. Phys. Fluids 26, 015114.

Paoletti, M. S. \& Lathrop, D. P. 2011 Angular momentum transport in turbulent flow between independently rotating cylinders. Phys. Rev. Lett. 106, 24501.

Pirró, D. \& QuAdrio, M. 2008 Direct numerical simulation of turbulent Taylor-Couette flow. Eur. J. Mech. B/Fluids 27, 552-566.

Racina, A. \& Kind, M. 2006 Specific power input and local micro mixing times in turbulent Taylor-Couette flow. Exp. Fluids 41, 513-522.

Ravelet, F., Delfos, R. \& Westerweel, J. 2010 Influence of global rotation and Reynolds number on the large-scale features of a Taylor-Couette flow. Phys. Fluids 22, 55103.

RiECKE, H. \& PAAP, H.-G. 1986 Stability and wave-vector restriction of axisymmetric Taylor vortex flow. Phys. Rev. A 33 (1), 547.

SNyder, H. A. 1969 Wave-number selection at finite amplitude in rotating Couette flow. J. Fluid Mech. 35 (2), 273-298.

Stuart, J. T. 1958 On the non-linear mechanics of hydrodynamic stability. J. Fluid Mech. $4(1), 1-21$.

TAKEDA, Y. 1999 Quasi-periodic state and transition to turbulence in a rotating Couette system. J. Fluid Mech. 389, 81-99.

TAYlOR, G. I. 1923 Stability of a viscous liquid contained between two rotating cylinders. Phil. Trans. R. Soc. Lond. A 223, 289-343.

TAYlOR, G. I. 1936 Fluid fraction between rotating cylinders. I. torque measurements. Proc. R. Soc. Lond. A 157, 546-564.

Tokgoz, S., Elsinga, G. E., Delfos, R. \& Westerweel, J. 2012 Spatial resolution and dissipation rate estimation in Taylor-Couette flow for tomographic PIV. Exp. Fluids 53, $561-583$.

van Gils, D. P. M., Huisman, S. G., Bruggert, G.-W., Sun, C. \& Lohse, D. 2011 Torque scaling in turbulent Taylor-Couette flow with co- and counterrotating cylinders. Phys. Rev. Lett. 106, 24502.

WENDT, F. 1933 Turbulente strömungen zwischen zwei rotierenden konaxialen zylindern. Ing.Arch. 4, 577-595.

XiaO, Q., Lim, T. T. \& Chew, Y. T. 2002 Effect of acceleration on the wavy Taylor vortex flow. Exp. Fluids 32, 639-644. 